\title{
Mining and characterization of ubiquitin E3 ligases expressed in the mouse testis
}

\author{
Xiaojun Hou ${ }^{1,2+}$, Wei Zhang ${ }^{1,2+}$, Zhenyu Xiao ${ }^{1,2}$, Haiyun Gan ${ }^{1}$, Xiwen Lin', Shangying Liao ${ }^{1}$ and Chunsheng Han ${ }^{1 *}$
}

\begin{abstract}
Background: Ubiquitin-mediated protein modification and degradation are believed to play important roles in mammalian spermatogenesis. The catalogues of ubiquitin activating enzymes, conjugating enzymes, and ligases (E3s) have been known for mammals such as mice and humans. However, a systematic characterization of E3s expressed during spermatogenesis has not been carried out.

Results: In present study, we set out to mine E3s from the mouse genome and to characterize their expression pattern, subcellular localization, and enzymatic activities based on microarray data and biochemical assays. We identified 398 putative E3s belonging to the RING, U-box, and HECT subfamilies and found that most genes were conserved between mice and humans. We discovered that 73 of them were highly or specifically expressed in the testes based on the microarray expression data. We selected 10 putative E3 genes to examine their mRNA expression pattern, and several genes to study their subcellular localization and E3 ligase activity. RT-PCR results showed that all the selected genes were predominately expressed in the testis. Some putative E3s were localized in the cytoplasm while others were in both the cytoplasm and the nucleus. Moreover, all the selected proteins were enzymatically active as demonstrated by in vitro and in vivo assays.

Conclusions: We have identified a large number of putative E3s that are expressed during mouse spermatogenesis. Among these, a significant portion is highly or specifically expressed in the testis. Subcellular localization and enzymatic activity assays suggested that these E3s might execute diverse functions in mammalian spermatogenesis. Our results may serve as an initial guide to the field for further functional analysis.
\end{abstract}

Keywords: Spermatogenesis, E3 ubiquitin ligase, Gene mining, Microarray, Testis

\section{Background}

Spermatogenesis, a complex yet highly regulated developmental process of male germ cells, consists of three stages--the proliferation of spermatogonia, the meiosis of spermatocytes and the morphogenesis of spermatids [1,2]. The formation of the terminally-differentiated germ cells, the spermatozoa, requires a series of important processes that are unique to spermatogenic cells, including nuclear condensation, mitochondrial rearrangement, histone replacement by transition proteins and protamin, the shedding of residual bodies, and the formation of acrosomes [3]. It is not surprising that a large number of testisspecific genes are required for these events. In mice and

\footnotetext{
* Correspondence: hancs@ioz.ac.cn

${ }^{\dagger}$ Equal contributors

'State Key Laboratory of Reproductive Biology, Institute of Zoology, Chinese Academy of Sciences, Beijing 100101, China

Full list of author information is available at the end of the article
}

rats, several high throughput gene expression datasets have revealed that a large number of genes are expressed to fuel spermatogenesis $[4,5]$. It is also estimated that about $4 \%$ of protein-coding genes in the mouse genome are specifically expressed in the mouse testis [6].

Ubiquitination of protein is an indispensable posttranslational modification that serves as a component of the protein quality control system. It is also involved in diverse biological processes such as signal transduction, DNA repair, transcriptional regulation in a protein degradation-independent way [7]. In principle, ubiquitination is a process containing three ubiquitin transferring reactions catalyzed by three corresponding enzymes. At first, the $8.5-\mathrm{kDa}$ ubiquitin polypeptide is activated by the ubiquitin-activating enzyme (E1) in an ATP-dependent way. Subsequently, this activated ubiquitin is transferred to the ubiquitin conjugating enzyme (E2) via a thioester bond. In the end, the ubiquitin is

\section{Biomed Central}


transferred to a lysine residue of the substrate catalyzed by the ubiquitin-protein ligase (E3) [8]. Substrate specificity is conferred by E3s, implying a much larger number of E3s in the genome than E1s and E2s. The mammalian genome encodes $1 \sim 2 \mathrm{E} 1 \mathrm{~s}, 10 \sim 20 \mathrm{E} 2 \mathrm{~s}$ and several hundred E3s [9]. There are three typical E3 families including the RING finger family, HECT domain family and the U-box family [10-12].

To our knowledge, approximate 29 E3s have been reported to be expressed in mammalian testes and most of them execute diverse functions at different stages of spermatogenesis (summarized in Additional file 1). A dozen of E3s as well as associated complex play important roles in DNA double strands break (DSB) and histone modifications during early meiosis. As examples, the expression of LASU1 complex and Cul4A-CRL4 is detected primarily in spermatogonia stage and involved in histone ubiquitination during meiosis [13-16]. An Nend rule pathway E3 ligase, UBR2, plays a critical role in transcriptional chromosome inactivation via ubiquitination of histone H2A [17]. The majority of E3s that are highly expressed in haploid germ cells probably contribute to the morphogenesis during the formation of spermatozoa. For instance, MARCH10 is a microtubule associated E3 and is involved in the organization and maintenance of the flagella of spermatozoa [18].

Although it has been known that the mammalian genome encodes a large number of E3s, the exact number is still not known particularly when higher specificity of mining is a major concern to the experimental scientists. Moreover, how these putative E3s are expressed during mammalian spermatogenesis is still an open question. In the present study, we mined out putative E3s from the mouse and human genome, evaluated their expression in multiple tissues, particularly in the mouse testis at different stages. The ligase activity of selected E3s was confirmed by in vitro and in vivo assays. Our list of E3s expressed during spermatogenesis provides a valuable source for future functional studies of the ubiquitination during mammalian spermatogenesis.

\section{Results}

\section{Mining of E3s from the mouse and human genomes}

To mine putative E3s from the mouse and human genomes, we first compiled all protein-coding genes from several microarray datasets (GSE96, GSE97, GSE1133, GSE2361,GSE9954, GSE1986) generated from gene expression profiling of multiple tissues and the EST dataset from the UniGene database [19-22]. As a result, 26762 and 23058 mouse and human genes were identified, respectively, and 15952 genes were homologous genes (47.1\%) based on the HomoloGene database annotation (Figure 1A). We then searched all protein coding genes for domains/motifs in the Pfam_ls and Pfam_fs library

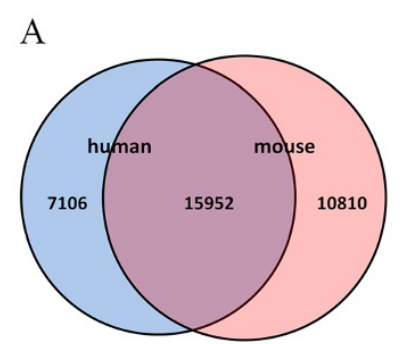

B

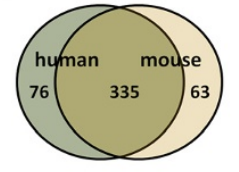

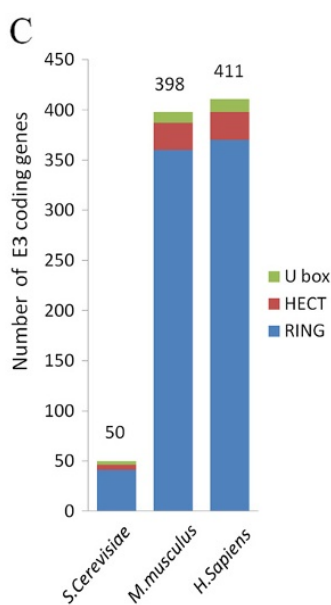

Figure 1 Numbers of putative E3s mined from the mouse and human genomes and the homologous genes between the two species. Gene list of protein-coding genes was compiled from lists of genes that can be detected using the Affymetrix microarray technology. Sequences of these genes were downloaded from NCBI Refseq database and were scanned for RING/U-box/HECT domains using the HMMER software. Homologue identification was based on the annotation of NCBI HomoloGene database. (A) Number of homologues of all protein-coding genes in humans and mice. The number in the intersection is the number of homologues while the other two represent genes unique to the two species. (B) Number of homologues of putative E3 genes in humans and mice. (C) Numbers of different $\mathrm{E} 3$ genes in humans, mice and yeasts.

(release 23.0) using HMMER 2.3.2 software package (http://hmmer.janelia.org/) with most of the parameters being set to the default values. The $\mathrm{E}$ value of the hit was set to be no more than 0.1. Proteins containing the RING domain (zf-C3HC4), the HECT domain and the U-box domain were considered as typical E3s. As a result, 398 and 411 putative E3s were identified from the mouse and the human genome, respectively (see Additional file 2 for mouse E3s). Among them, 335 putative E3s were homologues $(70.7 \%)$ between the two species (Figure 1B, Additional file 3). The proportion of homologues in the E3 set is significantly higher than expected from the number of general homologues of the mouse and the human genomes (P-value is $3.8 \mathrm{E}-29$ based on the binomial test). We also identified the yeast homologues of the initial 335 mouse/human homologous pairs from 50 yeast E3s (Figure $1 \mathrm{C}$ ), and only 7 highly conserved E3s among these three species were found (Table 1). These observations suggested that E3 genes are more conserved between the mouse and human genome.

\section{Expression of E3 mRNAs in the mouse testis}

We next examined the mRNA expression levels of E3 genes in the mouse based on four microarray datasets (GSE97, GSE1133, GSE9954, and GSE1986) and the UniGene dataset. For each dataset, we defined 5 expression 
Table 1 E3s conserved in the yeast, the mouse and the human

\begin{tabular}{|c|c|c|c|c|c|c|c|c|}
\hline \multicolumn{3}{|c|}{ Human } & \multicolumn{3}{|c|}{ Mouse } & \multicolumn{3}{|c|}{ Yeast } \\
\hline GeneID & Symbol & Chr. & GeneID & symbol & Chr. & GenelD & Symbol & Chr. \\
\hline 10277 & UBE4B & 1 & 63958 & Ube4b & 4 & 851337 & UFD2 & IV \\
\hline 4331 & MNAT1 & 14 & 17420 & Mnat1 & 12 & 852071 & TFB3 & IV \\
\hline 9921 & RNF10 & 12 & 50849 & Rnf10 & 5 & 851147 & MAG2 & $X \|$ \\
\hline 56852 & RAD18 & 3 & 58186 & Rad18 & 6 & 850430 & RAD18 & III \\
\hline 8315 & BRAP & 12 & 72399 & Brap & 5 & 856376 & ETP1 & VIII \\
\hline 5192 & PEX10 & 1 & 668173 & Pex10 & 4 & 851858 & PEX10 & IV \\
\hline 23327 & NEDD4L & 18 & 83814 & Nedd4I & 18 & 856862 & RSP5 & V \\
\hline
\end{tabular}

levels of an mRNA in a particular tissue in the increasing order of tissue specificity-A (absent), P (present), HP (highly present), MS (multiple tissue-specific), and SP (specific). The level $\mathrm{A} / \mathrm{P}$ is just based on a gene's $\mathrm{P} / \mathrm{A}$ call value determined by the Affymatrix platform using the MAS5 algorithm [23]. The other three levels were based on the comparison of the mRNA's z-score with three threshold values (See Methods for further explanation). The evaluations from different datasets were summarized by voting-A level value is assigned only when it is supported by no less than two datasets. A putative E3 is always assigned to the highest specificity group exclusively. As a result, we identified 267 P level E3s, 39 HP level E3s, 2 MS level E3s, and 32 SP level E3s in the mouse testis (Table 2). Compared with other tissues such as the liver, the lung, the muscle, the testis contains significantly more specific E3s (Figure 2). Therefore, a large number of E3s are expressed in the mouse testis and a significant portion is specifically expressed.

\section{Stage-specific expression of E3 mRNAs during mouse spermatogenesis}

We further studied the stage-specific expression of putative E3s during mouse spermatogenesis using three microarray datasets (GSE640, GSE926 and GSE4193) derived from gene expression profiling of testis at different days postpartum or of different spermatogenic cell types $[6,24,25]$. Genes that are 3-folds or more differentially expressed at one or more time point (or cell type) were selected for further clustering analysis. Based on visual inspection of the heat maps of the clustering analysis, three clusters of genes were identified according to the stages

Table 2 Numbers and mRNA expression levels of annotated E3s in the mouse testis

\begin{tabular}{lllllll}
\hline & SP & MS & HP & P & A & Total \\
\hline zf-C3HC4 & 30 & 2 & 33 & 237 & 58 & 360 \\
HECT & 0 & 0 & 5 & 22 & 0 & 27 \\
U box & 2 & 0 & 1 & 8 & 0 & 11 \\
total & 32 & 2 & 39 & 267 & 58 & 398 \\
\hline
\end{tabular}

when their expressions start to increase. Genes in group MI are expressed at a higher level at the mitotic division stage, genes in group ME start to increase their expression level at meiotic division stage while genes in group PM start to increase their expression at the post meiosis stage. Results from the three datasets were again summarized by voting-a gene was assigned to a particular group only if it was supported by 2 or 3 votes. As results, 20, 24 and 6 E3s were assigned to the MI, ME, and PM groups, respectively, totaling up to 50 (Table 3 and Additional file 4). By taking intersections of each of three groups with each of the SP, MS, HP, and P classes, it can be seen that the putative testis specific E3s increase their expression during or after meiosis.

\section{Stage-specific expression of E3 proteins during mouse spermatogenesis}

In a separate study, we examined protein levels in type A spermatogonia, pachytene spermatocytes, round spermatids, and elongated spermatids using the iTRAQ mass spectrometry (unpublished data). Thousands of proteins were identified in each cell type. We selected E3s from these detected proteins and examined their dynamic changes during spermatogenesis. 38 annotated E3s were found (Additional file 5). The heat maps of the clustering analysis showed that these proteins could be separated into two groups according to the dynamic change of their expression. Protein expression of group 1 is up regulated after meiosis while group 2 is on the contrary. Meanwhile, we performed an RNA deep sequencing in the same cell type (unpublished data). Compared to iTRAQ analysis, the expression patterns of most RNAs were consistent with those of protein expression. The changes of a portion of proteins were lagging behind those of their RNAs probably due to translation inhibition (Figure 3).

\section{Validation of mRNA expression of selected putative E3s by RT-PCR}

Among the 32 putative testis-specific E3s, 19 have not been well studied. We selected 10 of them randomly and 

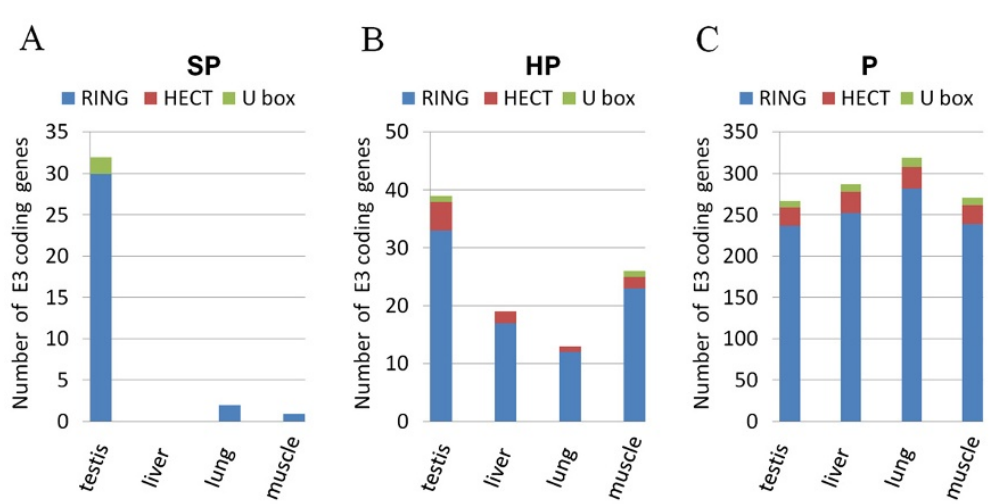

Figure 2 Expression profiles of E3 genes in different tissues. Expression of different families of E3s were ranked based on their levels in the mouse testis (P for present, HP for highly present, SP for specific). The expression of genes of these three levels were examined in other tissues such as the liver, the lung, and muscle. (A) Almost all the SP-level E3 genes were only expressed in the mouse testes. (B) More HP-level genes were expressed in the testes than in other tissues. (C) Genes that were expressed in the testis (P-level genes) were also expressed in other tissues.

checked their mRNA expression in 10 different tissues (testis, ovary, uterus, liver, lung, muscle, heart, brain, spleen and kidney) using RT-PCR. As shown by Figure 4A, majority were exclusively detected in the testis while the remaining one were expressed in the testis at much higher levels than in other tissues. These result indicated that our testis specificity evaluation of genes based on microarray data is highly reliable. Examination of their expression at different days postpartum indicated that almost all of them were expressed at stages when haploid cells are generated (Figure 4B). We further examined expression of some genes in isolated type A spermatogonia (SG-A), pachytene spermatocyte (pacSCs), round spermatid (rSTs) and elonged spermatid (eSTs). The purity of the isolated germ cells all exceeded $90 \%$ as determined by morphological evaluation, and was also confirmed by measuring the expression of known marker genes that are either uniquely or highly expressed in each type cells (Gfra 1 and Sohlh1 for type A spermatogonia; $L d h c$ for germ cell except spermatogonia; Sycp1, Piwil2 and H1t for spermatocytes; Tnp1 and Prm2 for spermatids; Fshr for sertoli cell). As shown by Figure $4 \mathrm{C}$, most of the E3s start their expression in either pacSCs or rSTs.

Table 3 Numbers of stage-specifically expressed E3 mRNAs during mouse spermatogenesis

\begin{tabular}{cccccc}
\hline $\begin{array}{c}\text { Cell } \\
\text { type }\end{array}$ & \multicolumn{4}{c}{ Testis expression level } & Total \\
\cline { 2 - 5 } & SP & MS & HP & P & \\
\hline MI & 0 & 0 & 2 & 18 & 20 \\
ME & 13 & 0 & 7 & 4 & 24 \\
PM & 5 & 0 & 0 & 1 & 6 \\
& 18 & 0 & 9 & 23 & 50 \\
\hline
\end{tabular}

Subcellular localization of putative E3s with or without transmembrane domains

A significant number of E3s have signal peptides and/or transmembrane domains (TMD) suggesting that they may anchor to the cell's membrane system to execute their functions. 16 and 65 annotated E3s were identified with either signal peptide and/or TMD, respectively (Figure 5A). As subcellular localizations of E3s help to understand their function, and no antibodies were available for in vivo studies, we decided to transfect $\mathrm{CHO}$ cells with plasmid constructs of E3s fused to EGFP to study their subcellular localization. We selected 2 E3s with TMD (RNF133, ZNRF4) and 3 without (RNF151, RNF126, NSMCE1), and used mouse Ubc6, an endoplasmic reticulum-localized integral membrane $\mathrm{E} 2$, as the reference [26]. CHO cells were co-transfected with the EGFP-E3 and the MmUbc6-DsRed constructs. Subcellular localization of the fusion proteins were inspected under fluorescent microscope. As shown in Figure 5B, RNF151 was only localized in the nucleus in a punctate manner. RNF126 and NSMCE1 was localized both in the nucleus and the cytoplasm in a diffused manner, while RNF133 and ZNRF4 were only localized in the cytoplasm, co-localized well with MmUbc6-DsRed. The localizations of RNF133 and ZNRF4 were consistent with previous report $[27,28]$, indicating that our assays using the fluorescent fusion proteins were reliable for subcellular localization studies.

\section{In vitro and in vivo ubiquitin ligase activities of putative E3s}

It was important to test whether the putative E3s indeed possess ubiquitin ligase activities in order to understand their function. However, due to the lack of antibodies to immunoprecipitate native E3s from testis or germ cell lysate, we decided to used recombinant protein and 


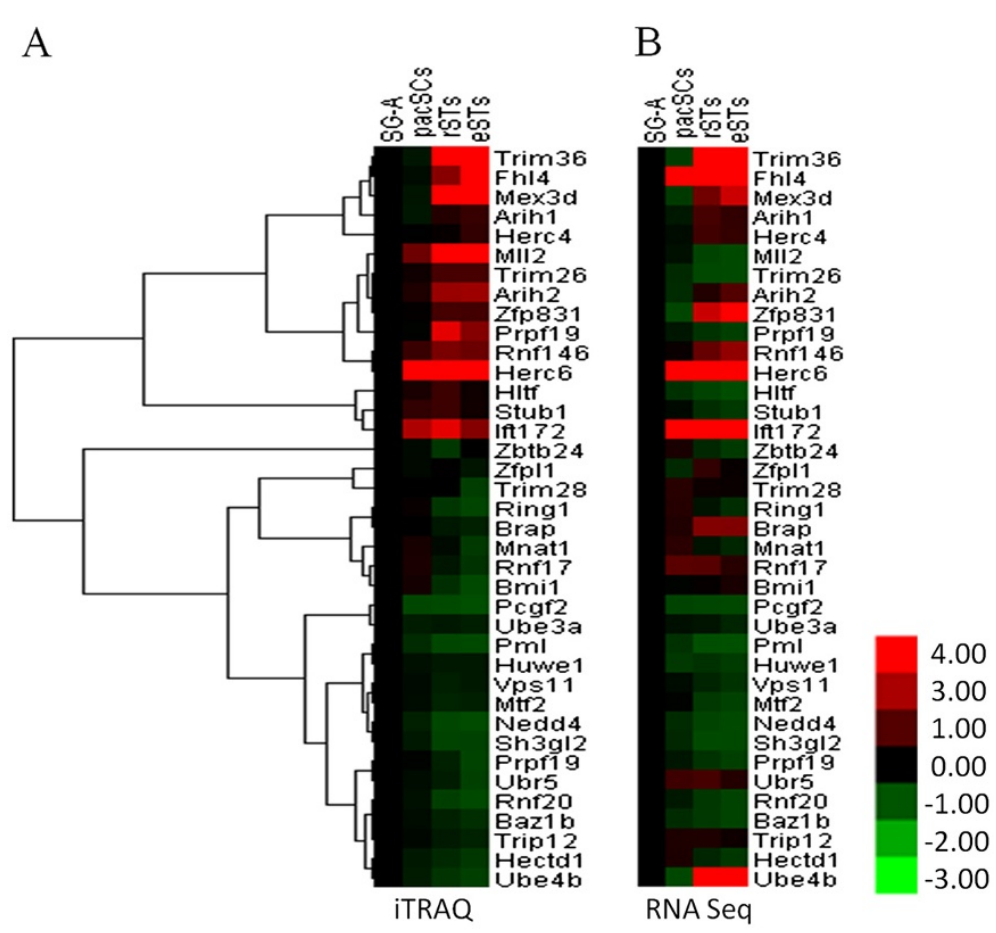

Figure 3 Putative E3s whose proteins were detected in one or more germ cell types using the iTRAQ mass spectrometry and their corresponding RNA levels detected by RNA-seq data. The protein and RNA expression data of these putative E3s were from two independent studies aiming to examine global gene expression at the mRNA and protein levels. Note most putative E3s were expressed at or after meiosis. The mRNA expression either matches or precedes their protein expression. SG-A: type A spermatogonia, pacSC: pachytene spermatocyte, rST: round spermatid, eST: elonged spermatid.

tagged protein immunoprecipitated from transfected cells to examine their enzymatic activity. Three putative E3 genes (Nsmce1, Rnf126 and Rnf151) were cloned into the pGEX-4T1 vector. GST fusion proteins were expressed and purified from $E$ coli. In vitro ubiquitination assays were performed by including E1, E2, E3, and ubiquitin. Anti-Ubiquitin (Ub) antibody was used to detect polyubiquitin chains. To screen the E2 with highest

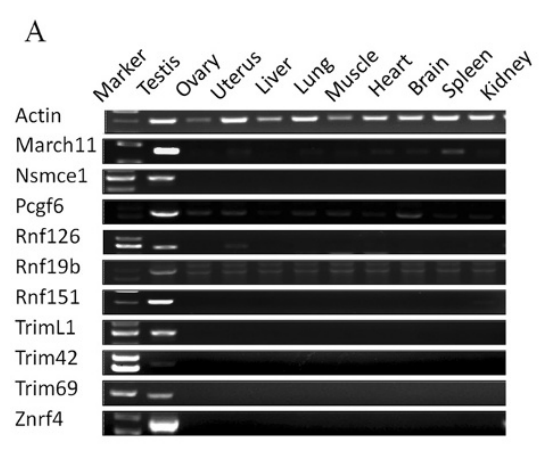

B

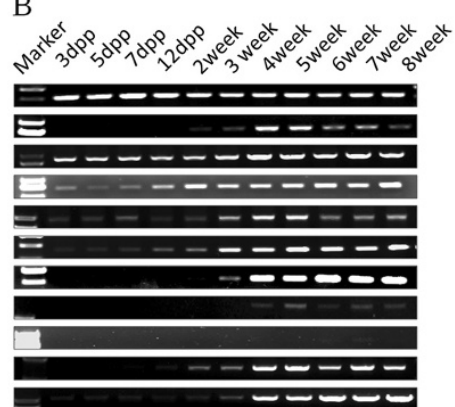

C

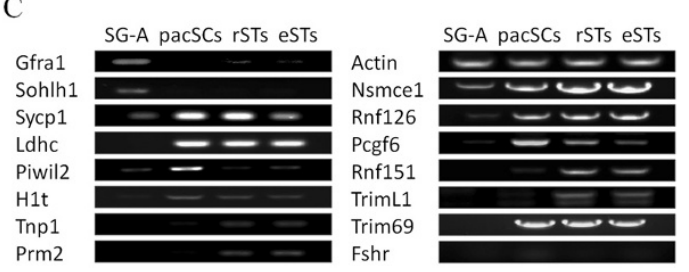

Figure 4 Examination of mRNA expression of annotated murine testis-specific E3s by RT-PCR. (A). mRNA expression in 10 tissues. (B). mRNA expression in testis of different days post partum. (C). mRNA expression in 4 isolated germ cell types. 


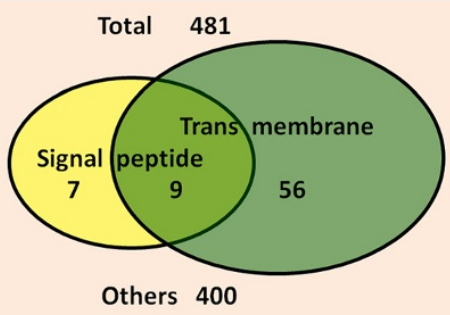

RNF151

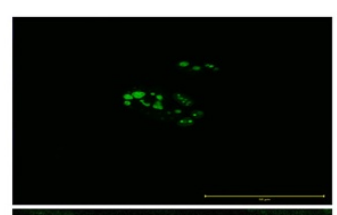

MmUbc6-DsRed
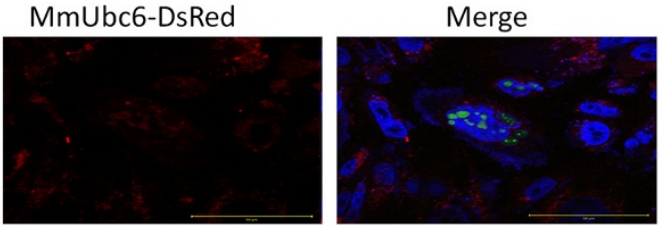

ZNRF4
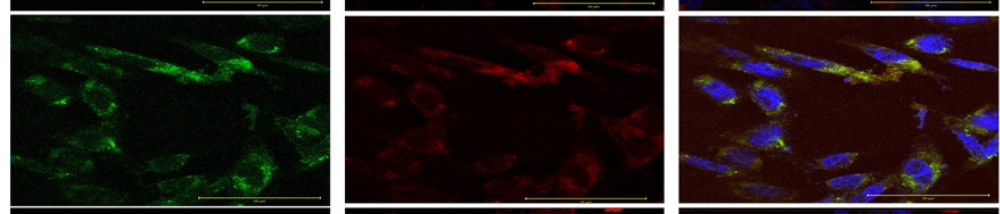

B

RNF126
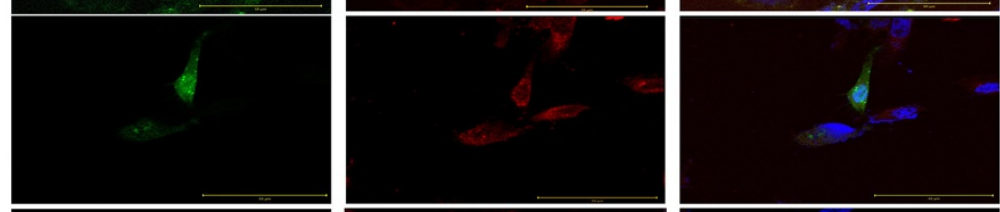

RNF133
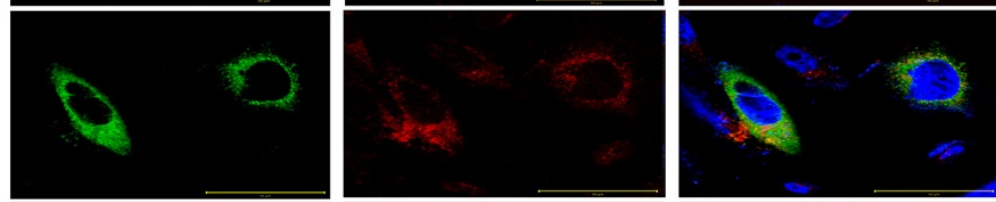

NSMCE1
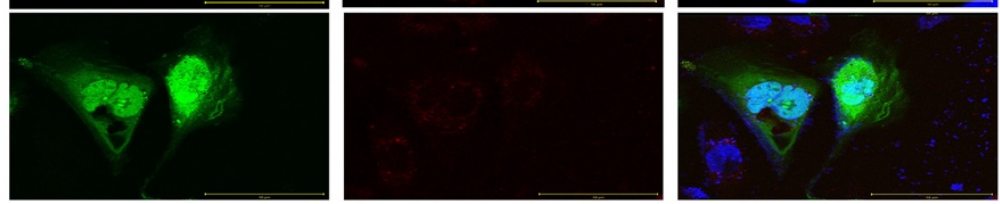

Figure 5 Analysis of E3s containing signal peptide and transmembrane domains (TMDs). (A) Number of E3s which possessed signal peptides and TMDs. (B) CHO cells were transiently transfected with GFP tag recombinant plasmids. The cells were then processed for immunofluorescence microscopy 24 hours after transfection and MG-132 treatment. The scale bar is $50 \mu \mathrm{m}$.

ubiquitin catalytic activity, three human and one mouse recombinant E2s (UBE2D2/human, UBE2D3/human, UBE2L3/human and Ube2k/mouse) were tested. Our results showed that UBE2L3 has barely-detectable activity while UBE2D2 has the highest activity (Figure 6). The polyubiquitin chain was formed when all reactants were added (E1, E2, E3, and ubiquitin), while, it was not detected if any of the reactants were excluded. Therefore, the ligase activity of the putative E3s was detected in a substrate-independent way.

The ligase activity of these E3s was also confirmed in HEK 293FT cells transfected with plasmid constructs that gave rise to FLAG-tagged E3s and HA-tagged Ub. Cell lysate was subjected to immunoprecipitation (IP) using the FLAG antibody conjugated beads and immunoblotted using the anti-HA antibody. As shown in
Figure 7A, poly (Ub) chains were detected for all tested E3s. It has been known that some E3s are first polyubiquitinated before they transfer the poly (Ub) chain to their substrates. To tell whether the poly (Ub) chain detected was from the E3s or any protein that was coimmunoprecipitated with the E3s, the cell lysate was first boiled with high concentration SDS to disrupt any potential protein-protein interaction before immunoprecipitated with the beads. As shown in Figure 7B, polyubiquitination was still detected with RNF126 and RNF151. These results showed that some E3s promoted self-ubiquitination before their substrates were ubiquitinated. A potential role of self-ubiquitination of E3s is to regulate the ligase activity and recruit substrates during spermatogenesis [29]. Our results also indicated that it was very likely that most, if not all, E3s identified by 


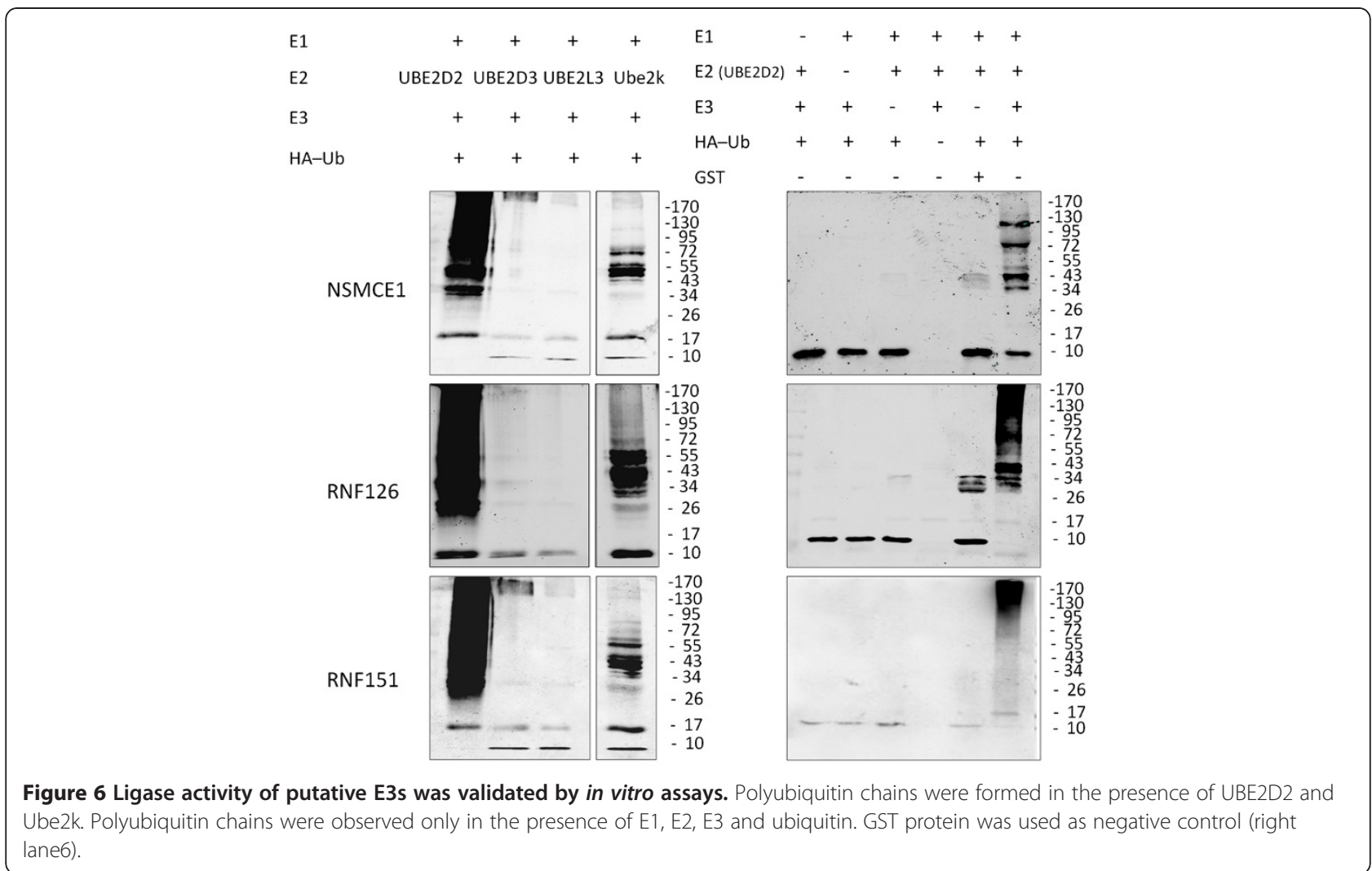

bioinformatics analysis were active enzymes. Considering their different sub-cellular localizations as indicated by the transfection assays, it seemed that these E3s were involved in diverse molecular processes during spermatogenesis such as protein quality control, organelle turnover, chromatin remodeling, to name a few.

\section{Discussion}

In the present study, we conducted genome-wide search and annotation of putative E3s from several highthroughput expression datasets. We mined out putative E3s from protein coding genes whose expression have been examined in different tissues, and investigated their mRNA and protein expressions in the mouse testis. According to microarray data, we discovered that a large number of putative E3s were expressed in the mouse testis and that some of them were specifically expressed there, which were confirmed by RT-PCRs. The protein expression patterns of $38 \mathrm{E} 3 \mathrm{~s}$ were detected in different spermatogenic cells using the iTRAQ MS technology (our unpublished data). The E3 ligase activity of some selected proteins was confirmed by ubiquitin assays. These results indicated that our mining and annotation strategy was reliable and distinguished its self from some previous mining procedures purely based on sequence analysis.
According to a report by $\mathrm{Li}$ et al. based on bioinformatics analysis, the human genome contains 617 putative E3s [9]. This number was derived from motif searching using the Hidden Markov Models (HMMs) for domains such as HECT, RING/U-box, F-box, SOCS-box etc. It is known that most, if not all, HECT and RING/ U-box proteins are true E3s while those F-box-, SOCSbox containing ones are not necessarily a component of the E3 complexes [30]. Therefore, we limited our searches by using only the HECT and RING/U-box domains to reduce the false positive rate. Indeed, $\mathrm{Li}$ et al. used several datasets containing predicted transcripts and corresponding proteins of the human genome in order for the search to be comprehensive. However, we think for bench-working scientists, higher specificity is more important than higher sensitivity. Accordingly, we only used protein-coding genes derived from the Affymetrix expression GeneChips (Mouse Genome 4302.0 Array, Murine Genome U74 Version 2 Array, Human Genome U95A Array, Human Genome U133A Array and GNF1M/GNF1H custom array) and the UniGene database as the target sets to be mined. As a result, we identified 398 putative E3s, of which 360 were the RING family members from the mouse genome. Our 411 human E3s are not all covered by Li's 617 E3s. The intersection between our 411 human E3s and the 617 by $\mathrm{Li}$ et al. is 308 . We found that the list of $\mathrm{Li}$ 
et al. only contained 343 human E3s based on the HECT and RING/U-box domains. Therefore, our mining should be more specific than Li et al. and also more sensitive if the same set of domains were used. Moreover, we found that Li et al. mined 34 more E3s based on only RING and U-box domains than we did. However, a manual curation of these 34 genes by us indicated that 26 actually did not contain the RING or U-box, 3 were pseudogenes, 1 was discontinued gene, 3 were E4s, and only 1 typical E3 was left.

The main goal of the present study was to profile the expression of E3s in the mouse testis during spermatogenesis. Taking advantage of several multiple tissue microarray datasets, we rated the expression levels of putative E3s as 5 levels (A, P, HP, MS and SP). The rating system was based on a supposed normal distribution of gene expression in different tissues and the final rate was determined by votes from different datasets. Therefore, the rating was highly stringent as was confirmed by the RT-PCR results. Almost all predicted testis-specific E3s were indeed specifically expressed in the testis. In addition, we found that majority of the putative E3s were expressed in the mouse testis, and the number of testisspecific E3s was much higher than in other tissues such as the liver, the lung, and the muscle. The stages-specific expression of E3s during mouse spermatogenesis indicated that most of the testis-specific E3s were expressed in spermatocytes and spermatids. As spermatocytes undergo meiosis and spermatids go through a number of unique processes such as acrosome formation, histone replacement, flagellum formation and shedding of most of the cytoplasm, it is reasonable that these cells need many tissue-specific E3s to catalyze the turnover of proteins.

\section{Conclusions}

We performed a mining of ubiquitin ligases in the mouse genome and analyzed their expression profile in 
the mouse testis systematically. The identification of a large number of homologous putative E3s between human and mouse suggests that these enzymes are highly conserved in the two species. Our analysis reveals that 340 putative E3s are expressed in the mouse testis and 73 E3s are highly or specifically expressed. Based on in vitro and in vivo essays, the ligase activity of several novel E3s expressed during the mouse spermatogenesis are confirmed. The lists of E3s expressed at different levels during spermatogenesis provide a valuable resource from which key factors regulating spermatogenesis could be identified.

\section{Methods}

\section{mRNA expression evaluation in tissues}

In order to summarize the expression levels of a gene in a particular tissue (t) from different datasets (d), the expression value of a gene in a tissue $\left(x_{t}\right)$ were converted into the $z$-score $\left(z_{t}=\left(x_{t}-\mu\right) / s\right.$ where $\mu$ and $s$ are the mean and standard deviation of $x_{t}$ among tissues). Assuming the $z_{t}$ values of a gene in different tissues are normally distributed, three threshold values $\left(\mathrm{z}_{1}=1\right.$, $\left.z_{2}=1.96, z_{3}=2.58\right)$ mean that at least $68 \%, 95 \%, 99 \%$ of tissues have the absolute values of their $z_{t}$ scores to be less than these value, respectively. For example, if a gene in the testis has its $z_{t=t e s t i s} \geq 2.58$, we can say that the $\mathrm{z}_{\mathrm{t} \neq \text { testis }}$ values of this gene in at least $99.5 \%$ of the tissues should be less than $z_{t=\text { testis }}$ while $z_{t \neq \text { testis }}$ values in at most $0.5 \%$ of the other tissues may be equal to or more than $z_{t=\text { testis. }}$ The following five expression levels were defined accordingly. A for absent of a gene in $t$ if the original Affymetrix P/A call value is A. P for present if the Affymetrix $\mathrm{P} / \mathrm{A}$ call value is $\mathrm{P}$. HP for highly present with $z_{t} \geq z_{1}$. MS for multi-tissue specific with $z_{t} \geq z_{2}$ because a gene with such an expression value may only expressed in several tissues. SP for specific with $z_{t} \geq z_{3}$ for tissue $t$ and $z_{i} \leq z_{2}$ for all the other tissues $(i \neq t)$. The most specific expression level was assigned to a gene if it satisfying more than one level. For example, a gene considered SP must also satisfy the MS condition, but we labeled it as SP. We considered five datasets (including the four mouse microarray datasets and the UniGene EST dataset) as five voters. We determined the mRNA expression level by a ballot. The expression level that was supported by the highest number of votes was assigned to the gene in the specified tissue. However, if two expression levels were both supported by the highest number of votes, the less specific level was assigned. For example, MS was assigned to a gene if both MS and SP were supported by 2 votes.

\section{Animal use}

Male CD1 mice were purchased from Vital River Laboratories (Beijing, China) and maintained in the
Experiment Animal Center, Chinese Academy of Sciences. Animal use was approved by the Animal Research Committee of the Institute of Zoology, Chinese Academy of Sciences (Protocol No. 2004-35). Protocols for animal sacrifice and tissue harvesting were in accordance with the NIH Guide for the Care and Use of Laboratory Animals. All the mice were euthanized by cervical dislocation before tissue harvesting.

\section{Isolation of different type germ cells}

$8 \mathrm{dpp}$ and $17 \mathrm{dpp}$ mice were used for isolating type A spermatogonia (SG-A) and pachytene spermatocytes (pacSCs), respectively. Meanwhile, adult mice were used for isolating round spermatids (rSTs) and elonged spermatids (eSTs). The seminiferous tubules were isolated from decapsulated testes and digested into cell suspension with collagenase $\left(1 \mathrm{mg} / \mathrm{ml}, 2 \mathrm{~min}, 37^{\circ} \mathrm{C}\right.$, Gibco $)$ and trypsin $\left(0.25 \%, 5 \mathrm{~min}, 37^{\circ} \mathrm{C}\right.$, Gibco). The purity of four types of germ cells all exceeded $90 \%$ using the unit gravity sedimentation procedure in $2-4 \%$ BSA medium as described before [31-33]. The purity of cells was validated based on morphological evaluation and confirmed by RT-PCR of reported marker genes expressed in different germ cells.

\begin{tabular}{|c|c|c|}
\hline Name & Product length & Sequence $\left(5^{\prime}-3^{\prime}\right)$ \\
\hline \multirow[t]{2}{*}{ Action } & $480 \mathrm{bp}$ & Sense: ATATCGCTGCGCTGGTCGTC \\
\hline & & Antisense: ATATCGCTGCGCTGGTCGTC \\
\hline \multirow[t]{2}{*}{ March11 } & $321 \mathrm{bp}$ & Sense: ATGAGCGACGAGGGCAAAAAAC \\
\hline & & Antisense: CTACTGAACTTTCTCAACCAGT \\
\hline \multirow[t]{2}{*}{ Nsmce1 } & $830 \mathrm{bp}$ & Sense: ATATGGCTTCCGGCGTGATC \\
\hline & & Antisense: TTGTGCTGCCGGGTCCGTAA \\
\hline \multirow[t]{2}{*}{ Pcgf6 } & 698 bp & Sense: AAGCCTGCTGCTCCACA \\
\hline & & Antisense: TGACCCACCCACCTCAA \\
\hline \multirow[t]{2}{*}{ Rnf126 } & 924 bp & Sense: ATATGGCCGAGGCGTCGCCG \\
\hline & & Antisense: TTGGAGTTGCTTGTGGCG \\
\hline \multirow[t]{2}{*}{ Rnf19b } & $407 \mathrm{bp}$ & Sense: AGCGGAAGCCCTACAGA \\
\hline & & Antisense: CCACCACCAGCACCATT \\
\hline \multirow[t]{2}{*}{ Rnf151 } & $519 \mathrm{bp}$ & Sense: GCCGCCTACAAGTCAAG \\
\hline & & Antisense: GCCTGGGTTCAGTGTTA \\
\hline \multirow[t]{2}{*}{ TrimL1 } & $1850 \mathrm{bp}$ & Sense: TGATGTCCAACCATGAGA \\
\hline & & Antisense: CAGATGTGGCAGATGATAAG \\
\hline \multirow[t]{2}{*}{ Trim42 } & $816 \mathrm{bp}$ & Sense: ACCAATCTGAAGTGCTACTA \\
\hline & & Antisense: CTACAGGTCATTATCAAT \\
\hline \multirow[t]{2}{*}{ Trim69 } & $1517 \mathrm{bp}$ & Sense: CAAGTTTCATGGAGGTATC \\
\hline & & Antisense: ATCTGTGGATGTACGATGTG \\
\hline \multirow[t]{2}{*}{ Znrf4 } & 876 bp & Sense: CATGGCGCGGTTTGCGTG \\
\hline & & Antisense: TCAGGAGAGCTCGGAAGTCGCTTC \\
\hline
\end{tabular}




\section{RNA isolation and RT-PCR}

Total RNAs were extracted from mouse testes at different developmental stages and other tissues using TRIzol solution. $2 \mu \mathrm{g}$ RNA was reverse-transcribed into cDNA by reverse transcriptase (Promega). The primers used for PCR are listed in Table 4. PCR reactions were conducted following stand protocol. Briefly, the reactions were started at $94^{\circ} \mathrm{C}$ for $3 \mathrm{~min}$, and went through 27 cycles with denaturing at $94^{\circ} \mathrm{C}$ for $30 \mathrm{sec}$, annealing $60^{\circ} \mathrm{C}$ (or other suitable Tms) for $30 \mathrm{sec}$, and elongation at $72^{\circ} \mathrm{C}$ for $40 \mathrm{sec}$. Reactions were finished with a final extension step at $72^{\circ} \mathrm{C}$ for $10 \mathrm{~min}$.

\section{Molecular cloning of putative E3s}

cDNAs of putative mouse E3s were amplified using PCRs. Restriction enzyme sites were included in the primers. PCR products and vector plasmids were digested with selected restriction enzymes, target fragments were gel purified and ligated subsequently. Full- or partial length cDNAs of putative E3s were sub-cloned into pFLAG-CMV4, pEGFP-N1 and pGEX-4T1 to make different fusion proteins. The primers used for plasmid construction were listed in Table 5 . All the recombinant plasmids were confirmed by DNA sequencing. Cloning procedures followed standard protocols.

\section{Preparation of recombinant proteins}

Putative E3s with GST tag and E2s (UBE2D2, UBE2D3, UBE2L3, Ube2k) with His tag were produced in the Escherichia coli strain Rossetta (DE3) (TransGen). Bacterial culture, IPTG treatment and lysate sonication were conducted following standard protocols. GST and His tag fusion proteins were purified by $\mathrm{AKTA}^{\mathrm{TM}}$ purifier 9500 using GST-Trap_5ml_FF and His-Trap_5ml_FF columns (GE Health care), respectively. Protein purification followed the manufacturer's instructions. Purified proteins were dialyzed by ultrafiltration (MICROCON, Millipore) before in vitro ubiquitination assays were performed.

\section{In vitro ubiquitination assays}

In vitro ubiquitination reactions were set up by adding to the Eppendorf tubes with the following reactants: $0.25 \mu \mathrm{g}$ yeast E1 (Boston Biochem); $2 \mu \mathrm{g}$ various purified recombinant E2s (UBE2D2/UBCH5B, UBE2D3/UBCH5C, UBE2L3/ UBCH7, Ube2k/E2-25K); $5 \mu \mathrm{g}$ putative E3s with GST tag; $5 \mu \mathrm{g}$ MYC-ubiquitin (Boston Biochem) in $50 \mathrm{mM}$ Tris/HCl (pH 7.4), $5 \mathrm{mM} \mathrm{MgCl}_{2}, 1 \mathrm{mM}$ DTT; 2 mM ATP (Sigma). Negative control reaction was set up with putative E3 being replaced with equal amount of glutathione S-transferase. After $30 \mathrm{~min}$ incubation at $37^{\circ} \mathrm{C}$,

Table 5 Primers used for recombinant plasmids construction

\begin{tabular}{|c|c|c|}
\hline Name & Vector & Sequence (5'-3') \\
\hline \multirow[t]{6}{*}{ Nsmce1 } & pGEX-4 T1 & Sense: CG GGATCC ATGGCTTCCGGCGTGATC \\
\hline & & Antisense: CG GAATTC CTAGTGCTGCCGGGTCCGTAA \\
\hline & pEGFP-N1 & Sense: CG GAATTC ATATGGCTTCCGGCGTGATC \\
\hline & & Antisense: CG GGATCC TTGTGCTGCCGGGTCCGTAA \\
\hline & pFLAG-CMV4 & Sense: CG GAATTC TATGGCTTCCGGCGTGATC \\
\hline & & Antisense: CG GGATCC CTAGTGCTGCCGGGTCCGTAA \\
\hline \multirow[t]{6}{*}{ Rnf126 } & pGEX-4 T1 & Sense: CG GGATCC ATGGCCGAGGCGTCGCCG \\
\hline & & Antisense: CG GAATTC TCAGGAGTTGCTTGTGGCG \\
\hline & pEGFP-N1 & Sense: CG GAATTC ATATGGCCGAGGCGTCGCCG \\
\hline & & Antisense: CG GGATCC TTGGAGTTGCTTGTGGCG \\
\hline & pFLAG-CMV4 & Sense: CG GAATTC TATGGCCGAGGCGTCGCCG \\
\hline & & Antisense: CG GGATCC TCAGGAGTTGCTTGTGGCG \\
\hline \multirow[t]{4}{*}{ Rnf151 } & pGEX-4 T1 & Sense: CCG GGATCC TTCCTGTGTTCTGTCTGCCA \\
\hline & & Antisense: GC CTCGAG CCTTCATAAGACACTTTGGC \\
\hline & pFLAG-CMV4 & Sense: CG GAATTC GATC ATGAGTGGTGG \\
\hline & & Antisense: CG GGATCC TCATAAGACACTTTGGC \\
\hline \multirow[t]{4}{*}{ Znrf4 } & pEGFP-N1 & Sense: CCG GAATTC CCATGGCGCGGTTTGCGTG \\
\hline & & Antisense: CCG GGATCC CTGGAG AGCT CGGA AGT CGC TTC \\
\hline & pFLAG-CMV4 & Sense: CCG GAATTC CATGGCGCGGTTTGCGTG \\
\hline & & Antisense:CCG GGATCC TCAGGAGAGCTCGGAAGTCGC TTC \\
\hline
\end{tabular}


reactions were stopped by treatment at $95^{\circ} \mathrm{C}$ for $10 \mathrm{~min}$ in a SDS-PAGE sample buffer containing $\beta$-mercaptoethanol. Then proteins were separated by electrophoresis and subjected to Western blot using anti-ubiquitin antibody [34].

\section{Cell culture and transfection}

HEK 293FT and CHO cells were cultured in high glucose DMEM (Gibco) supplemented with $10 \%$ fetal bovine serum (Hyclone) in $5 \% \mathrm{CO}_{2}$ at $37^{\circ} \mathrm{C}$. 24 hours after passage, $3.5 \times 10^{4} / \mathrm{cm}^{2}$ cells were plated and transient transfected with Mega Trans1.0 (ORIGEN) according to the manufacturer's recommendations. 24 hours after transfection, CHO cells were treated with $10 \mu \mathrm{M}$ MG132 (Z-Leu-Leu -Leu-al, Sigma) for 6 8 hours before fixed in $4 \%$ paraformaldehyde for $15 \mathrm{~min}$ at room temperature. Then the cells were permeabilized with $0.1 \%$ Triton X-100 and washed by PBS. Cell nucleus was dyed with DAPI $(1 \mu \mathrm{g} / \mathrm{ml})$ for 10 minutes. Samples were observed with Zeiss LSM710 confocal microscope. All the images were quantified using the ZEN software (Zeiss).

\section{In vivo Ubiquitination assays}

HEK 293FT cells transfected with different FLAG-tagged putative E3s and HA-tagged PRK-Ub were cultured for 36 hours. Cells were treated with $10 \mu \mathrm{M}$ proteasome inhibitor MG-132 for 6 8 hours. Subsequently, the cells were washed twice in ice-cold PBS and suspended in cell lysis buffer $[50 \mathrm{mM}$ Tris $\mathrm{HCl}$ (pH7.4), $150 \mathrm{mM} \mathrm{NaCl}$, $1 \mathrm{mM}$ EDTA, 1\% NP-40, and $10 \mu \mathrm{M}$ protease Inhibitor Cocktail (Sigma)]. The cell lysate $\left(3 \times 10^{7}\right.$ cells $/ \mathrm{ml}$, approximately $0.5 \sim 1 \mathrm{mg} / \mathrm{ml}$ ) was incubated on a shaker for 30 minutes at $4^{\circ} \mathrm{C}$ and centrifuged at $15000 \mathrm{~g}$ for $15 \mathrm{~min} .1 \mathrm{ml}$ supernatant was incubated with $30 \mu \mathrm{l}$ suspended ANTI-FLAG M2 affinity gel (Sigma) overnight at $4^{\circ} \mathrm{C}$ and then spun at $15,000 \mathrm{~g}$ for $15 \mathrm{~min}$ [35]. The M2 resin was washed 4 times with buffer A [50 mM Tris- $\mathrm{HCl}$ (pH7.4), $150 \mathrm{mM} \mathrm{NaCl}$ ], bound proteins were eluted using buffer B (including $150 \mathrm{ng} / \mu \mathrm{l} 1 \times$ FLAG peptide, Sigma). Eluted proteins were separated by electrophoresis using 4 20\% gradient PAGE gels [36,37].

In order to disrupt the non-covalent interactions, cells were lysed with lysis buffer containing $1 \%(\mathrm{w} / \mathrm{v})$ sodium dodecyl sulfate (SDS) and then heated to $100^{\circ} \mathrm{C} .10 \mathrm{mM}$ sulfhydryl alkylating agent N-ethylmaleimide (NEM, Pierce) was added into the cell lysis buffer to prevent ubiquitin chain from breaking. The concentrations of SDS in the cell lysis were diluted to $0.1 \%$ before immunoprecipitated with the ANTI-FLAG M2 beads [38].

\section{Western blot analysis}

Western blot analysis followed standard protocol. Primary antibodies and dilution factors were as the following: anti-
FLAG and anti-HA, 1:5000 (mouse, monoclonal antibody, Abmart); anti-ubiquitin, 1:1000 (mouse, polyclonal antibody, Santa Cruz). The secondary antibody was antimouse IgG (goat polyclonal antibody labeled with Dylight 800, 1:5000, KPL). Fluorescent signal on NC-membrane was detected with an Odyssey infrared imaging system (LI-COR Biosciences).

\section{Additional files}

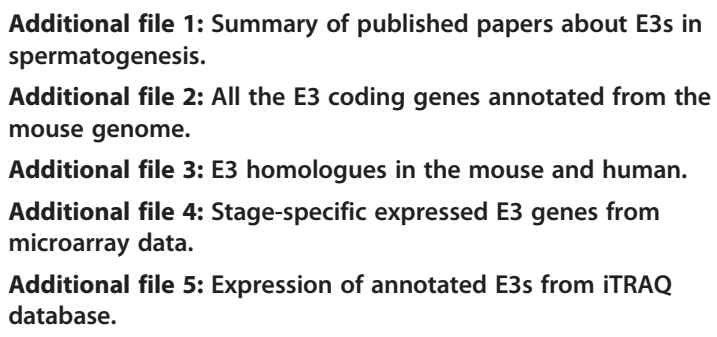

Competing interests

The authors declare that they have no competing interests.

\section{Authors' contributions}

$\mathrm{CH}, \mathrm{XH}$, and $\mathrm{WZ}$ conceived and designed the experiments. $\mathrm{XH}, \mathrm{WZ}, \mathrm{SL}$ and ZX conducted the experiments. WZ, HG and XL analyzed microarray data. $\mathrm{XH}$, and $\mathrm{CH}$ wrote the manuscript. All authors have read and approved the final manuscript.

\section{Acknowledgments}

We thank Dr. Wei Li from the Institute of Zoology, Chinese Academy of Sciences for providing us plasmids for making recombinant E2s. This study was supported by the National Basic Research Program of China (2006CB944004, 2012CB966702) and the National Natural Science Foundation of China (30871406).

\section{Author details}

${ }^{1}$ State Key Laboratory of Reproductive Biology, Institute of Zoology, Chinese Academy of Sciences, Beijing 100101, China. ${ }^{2}$ Graduate University of Chinese Academy of Sciences, Beijing 100049, China.

Received: 2 April 2012 Accepted: 7 September 2012

Published: 19 September 2012

\section{References}

1. Russell LD, Ettlin RA, Sinha HAP, Clegg ED: Mammalian spermatogenesis. In Histological and Histopathological Evaluation of the Testis. Edited by Russell LD, Ettlin RA, Sinha HAP, Clegg ED. St. Louis: Cache River Press; 1990:1-40.

2. Dym M: Spermatogonial stem cells of the testis. Proc Natl Acad Sci U S A 1994, 91(24):11287-11289.

3. Hecht NB: Molecular mechanisms of male germ cell differentiation. Bioessays 1998, 20(7):555-561.

4. Johnston DS, Wright WW, Dicandeloro P, Wilson E, Kopf GS, Jelinsky SA: Stage-specific gene expression is a fundamental characteristic of rat spermatogenic cells and Sertoli cells. Proc Natl Acad Sci U S A 2008, 105(24):8315-8320

5. Pang AL, Johnson W, Ravindranath N, Dym M, Rennert OM, Chan WY: Expression profiling of purified male germ cells: stage-specific expression patterns related to meiosis and postmeiotic development Physiol Genomics 2006, 24(2):75-85.

6. Schultz N, Hamra FK, Garbers DL: A multitude of genes expressed solely in meiotic or postmeiotic spermatogenic cells offers a myriad of contraceptive targets. Proc Natl Acad Sci U S A 2003, 100(21):12201-12206.

7. Welchman RL, Gordon C, Mayer RJ: Ubiquitin and ubiquitin-like proteins as multifunctional signals. Nat Rev Mol Cell Biol 2005, 6(8):599-609. 
8. Hershko A, Heller H, Elias S, Ciechanover A: Components of ubiquitinprotein ligase system. Resolution, affinity purification, and role in protein breakdown. J Biol Chem 1983, 258(13):8206-8214.

9. Li W, Bengtson MH, Ulbrich A, Matsuda A, Reddy VA, Orth A, Chanda SK, Batalov S, Joazeiro CA: Genome-wide and functional annotation of human E3 ubiquitin ligases identifies MULAN, a mitochondrial E3 that regulates the organelle's dynamics and signaling. PLoS One 2008, 3(1):e1487.

10. Lorick KL, Jensen JP, Fang S, Ong AM, Hatakeyama S, Weissman AM: RING fingers mediate ubiquitin-conjugating enzyme (E2)-dependent ubiquitination. Proc Natl Acad Sci U S A 1999, 96(20):11364-11369.

11. Huibregtse JM, Scheffner M, Beaudenon S, Howley PM: A family of proteins structurally and functionally related to the E6-AP ubiquitin-protein ligase. Proc Natl Acad Sci U S A 1995, 92(11):5249.

12. Aravind $\mathrm{L}$, Koonin $\mathrm{EV}$ : The $\mathrm{U}$ box is a modified RING finger - a common domain in ubiquitination. Curr Biol 2000, 10(4):R132-R134.

13. Liu Z, Oughtred R, Wing SS: Characterization of E3Histone, a novel testis ubiquitin protein ligase which ubiquitinates histones. Mol Cell Biol 2005, 25(7):2819-2831

14. Liu Z, Miao D, Xia Q, Hermo L, Wing SS: Regulated expression of the ubiquitin protein ligase, E3(Histone)/LASU1/Mule/ARF-BP1/HUWE1, during spermatogenesis. Dev Dyn 2007, 236(10):2889-2898.

15. Wang $H$, Zhai L, Xu J, Joo HY, Jackson S, Erdjument-Bromage H, Tempst $P$, Xiong $\mathrm{Y}$, Zhang $\mathrm{Y}$ : Histone $\mathrm{H} 3$ and $\mathrm{H} 4$ ubiquitylation by the CUL4-DDBROC1 ubiquitin ligase facilitates cellular response to DNA damage. Mol Cell 2006, 22(3):383-394.

16. Kopanja D, Roy N, Stoyanova T, Hess RA, Bagchi S, Raychaudhuri P: Cul4A is essential for spermatogenesis and male fertility. Dev Biol 2011, 352(2):278-287.

17. An JY, Kim EA, Jiang Y, Zakrzewska A, Kim DE, Lee MJ, Mook-Jung I, Zhang Y, Kwon YT: UBR2 mediates transcriptional silencing during spermatogenesis via histone ubiquitination. Proc Natl Acad Sci U S A 2010, 107(5):1912-1917.

18. Iyengar PV, Hirota T, Hirose $S$, Nakamura N: Membrane-associated RING-CH 10 (MARCH10 protein) is a microtubule-associated E3 ubiquitin ligase of the spermatid flagella. J Biol Chem 2011, 286(45):39082-39090.

19. Su Al, Cooke MP, Ching KA, Hakak Y, Walker JR, Wiltshire T, Orth AP, Vega RG, Sapinoso LM, Mogrich A, et al: Large-scale analysis of the human and mouse transcriptomes. Proc Natl Acad Sci U S A 2002, 99(7):4465-4470.

20. Su Al, Wiltshire T, Batalov S, Lapp H, Ching KA, Block D, Zhang J, Soden R, Hayakawa M, Kreiman $G$, et al: A gene atlas of the mouse and human protein-encoding transcriptomes. Proc Natl Acad Sci U S A 2004 , 101(16):6062-6067.

21. Ge X, Yamamoto S, Tsutsumi S, Midorikawa Y, Ihara S, Wang SM, Aburatani H: Interpreting expression profiles of cancers by genome-wide survey of breadth of expression in normal tissues. Genomics 2005, 86(2):127-141.

22. Thorrez L, Van Deun K, Tranchevent LC, Van Lommel L, Engelen K, Marchal K, Moreau Y, Van Mechelen I, Schuit F: Using ribosomal protein genes as reference: a tale of caution. PLoS One 2008, 3(3):e1854

23. Affymetrix: Microarray suite users guide. 5th edition. Santa Clara: Affymetrix; 2001

24. Shima JE, McLean DJ, McCarrey JR, Griswold MD: The murine testicular transcriptome: characterizing gene expression in the testis during the progression of spermatogenesis. Biol Reprod 2004, 71(1):319-330.

25. Namekawa SH, Park PJ, Zhang LF, Shima JE, McCarrey JR, Griswold MD, Lee JT: Postmeiotic sex chromatin in the male germline of mice. Curr Biol 2006, 16(7):660-667.

26. Tiwari S, Weissman AM: Endoplasmic reticulum (ER)-associated degradation of T cell receptor subunits. Involvement of ER-associated ubiquitinconjugating enzymes (E2s). J Biol Chem 2001, 276(19):16193-16200

27. Nian H, Zhang W, Shi H, Zhao Q, Xie Q, Liao S, Zhang Y, Zhang Z, Wang C, Han C: Mouse RING finger protein Rnf133 is a testis-specific endoplasmic reticulum-associated E3 ubiquitin ligase. Cell Res 2008, 18(7):800-802.

28. Neutzner A, Neutzner M, Benischke AS, Ryu SW, Frank S, Youle RJ, Karbowski M: A systematic search for endoplasmic reticulum (ER) membrane-associated RING finger proteins identifies Nixin/ZNRF4 as a regulator of calnexin stability and ER homeostasis. J Bio/ Chem 2011, 286(10):8633-8643.

29. de Bie P, Ciechanover A: Ubiquitination of E3 ligases: self-regulation of the ubiquitin system via proteolytic and non-proteolytic mechanisms. Cell Death Differ 2011, 18(9):1393-1402.

30. Petroski MD, Deshaies RJ: Function and regulation of cullin-RING ubiquitin ligases. Nat Rev Mol Cell Biol 2005, 6(1):9-20.
31. Romrell $L$, Bellve AR, Fawcett DW: Separation of mouse spermatogenic cells by sedimentation velocity. A morphological characterization. Dev Biol 1976, 49(1):119-131.

32. Bellve AR, Millette CF, Bhatnagar YM, O'Brien DA: Dissociation of the mouse testis and characterization of isolated spermatogenic cells. $J$ Histochem Cytochem 1977, 25(7):480-494.

33. Gan $H$, Lin $X$, Zhang Z, Zhang W, Liao S, Wang L, Han C: piRNA profiling during specific stages of mouse spermatogenesis. RNA 2011, 17(7):1191-1203.

34. Nishito $Y$, Hasegawa M, Inohara N, Nunez G: MEX is a testis-specific E3 ubiquitin ligase that promotes death receptor-induced apoptosis. Biochem J 2006, 396(3):411-417.

35. Ogawa M, Mizugishi K, Ishiguro A, Koyabu Y, Imai Y, Takahashi R, Mikoshiba K, Aruga J: Rines/RNF180, a novel RING finger gene-encoded product, is a membrane-bound ubiquitin ligase. Genes Cells 2008, 13(4):397-409.

36. Locke $\mathrm{M}$, Tinsley $\mathrm{CL}$, Benson MA, Blake DJ: TRIM32 is an E3 ubiquitin ligase for dysbindin. Hum Mol Genet 2009, 18(13):2344-2358.

37. Bonifacino JS, Dell'Angelica EC, Springer TA: Immunoprecipitation. Curr Protoc Immunol 2001, 8:8-3.

38. Laney JD, Hochstrasser M: Analysis of protein ubiquitination. Curr Protoc Protein Sci 2002, 14:14-15.

doi:10.1186/1471-2164-13-495

Cite this article as: Hou et al:: Mining and characterization of ubiquitin E3 ligases expressed in the mouse testis. BMC Genomics 2012 13:495.

\section{Submit your next manuscript to BioMed Central and take full advantage of:}

- Convenient online submission

- Thorough peer review

- No space constraints or color figure charges

- Immediate publication on acceptance

- Inclusion in PubMed, CAS, Scopus and Google Scholar

- Research which is freely available for redistribution 